\title{
Autonomy of migration: (de)territorialità, resistenza e diritto di cittadinanza nella letteratura della migrazione
}

\author{
Autonomy of Migration: (De)territorialization, \\ Resistance and Right of Citizenship in Migration Literature
}

\author{
VinCENZo BinetTi [vbinetti@umich.edu] \\ University of Michigan, Stati Uniti d'America
}

\begin{abstract}
Riassunto
L'elemento indubbiamente provocatorio che scaturisce dall'analisi delle storie narrate da scrittori migranti oggi in Italia è, a mio avviso, l'intenzione autoriale di voler in qualche modo decentralizzare e quasi sfocare la visualizzazione della territorialità all'interno della quale operano e si muovono i personaggi e le loro storie. Anche se spesso ambientati in contesti urbani e metropolitani all'apparenza facilmente riconoscibili ed identificabili dal lettore, manca infatti in questi romanzi un riferimento direi quasi rassicurante a quei landmarks architettonici e urbanistici che dovrebbero invece contraddistinguere e avvalorare emblematicamente i paesaggi e la mappatura della tradizione storico-culturale dell'identità nazionale italiana. Mi chiedo allora che tipo di implicazioni politico-letterarie possano scaturire da questa deliberata invisibilità dei landscapes ufficiali e da una esclusione dell'altro dai paesaggi urbani all'interno dei quali si muovono incerti ed esitanti questi cittadini stranieri protagonisti della scrittura migrante. Quali potenziali fattori destabilizzanti della canonicità letteraria e quali forme di resistenza politica emergono allora da questi tentativi di (ri)leggere l'uniformità impermeabile del territorio nazionale proprio spostando il punto di vista interpretativo di quegli spazi pubblici e quindi spiazzando l'orizzonte d'attesa di chi legge e che in quegli spazi vorrebbe riconoscere e validare in un qualche modo il proprio senso di appartenenza identitaria?
\end{abstract}

\section{Parole Chiave}

Amara Lakhous; Letteratura della migrazione; Paesaggio; Spazio urbano; Autonomia; Resistenza; Esclusione; Cittadinanza

\section{Abstract}

Most of the novels written by migrant writers today in Italy have, in my opinion, a tendency to purposely decentralize and almost blur the visualization of a given territoriality within which their characters evolve and operate. Even if these novels are, in fact, often set in metropolitan contexts easily recognizable and identifiable by the reader, they are indeed missing an almost reassuring reference to those urban and architectural landmarks, which should instead have emblematically validated and clearly demarcated the landscapes and the mapping of the Italian cultural-historical tradition and its national identity. I wonder therefore which kind of political and literary implications may spring from this deliberate invisibility of the official landscapes and from an exclusion of the other from the urban spaces within which these fo- 
reign citizens, nomadic protagonists of migrant narratives, erratically move. Which destabilizing literary and stylistic elements define then these attempts to (re)read the impermeable uniformity of the national territory through a problematic and conflictual shifting of the interpretative point of view of those same public spaces? And, even more, which forms of political resistance define this intentionality, this desire to displace a certain predictability of the reader's orizzonte d'attesa, a reader who would have preferred instead to recognize and validate, precisely within those spaces, his/her own sense of identitarian belonging and citizenship?

\title{
KEYWORDS
}

Amara Lakhous; Migration Literature; Landscape; Urban Space; Autonomy; Resistance; Exclusion; Citizenship

RICEVUTO 2015-08-31; ACCETTATO 2015-12-10

\begin{abstract}
"There was this Englishman who worked in the London office of a multicultural corporation based in the United States. He drove home one evening in his Japanese car. His wife, who worked in a firm, which imported German kitchen equipment, was already at home. Her small Italian car was often quicker through the traffic. After a meal which included New Zealand lamb, Californian carrots, Mexican honey, French cheese and Spanish wine, they settled down to watch a programme on their television set, which was made in Finland. The programme was about a retrospective celebration of the war to recapture the Falkland Islands. As they watched it, they felt very warmly patriotic, and very proud to be British."
\end{abstract}

Raymond Williams, Towards 2000

\begin{abstract}
"Nell'immaginario coloniale, il colonizzato non è soltanto un altro che è stato espulso dal regno della civiltà, ma è prodotto e percepito come l'Altro, come l'assoluta negazione situata all'estremo limite dell'orizzonte."
\end{abstract}

Negri e Hardt, Impero

Forse dovremmo iniziare il nostro discorso sulla geografia della letteratura migrante evidenziandone problematicamente proprio la sua assenza, il suo costituirsi cioè all'interno della trama quasi come un no-landscape, un no-lieu, per parafrasare l'intuizione di Marc Augé. Lelemento indubbiamente provocatorio che scaturisce dall'analisi delle storie narrate da scrittori migranti oggi in Italia è, a mio avviso, l'intenzione autoriale di voler in qualche modo decentralizzare e quasi sfocare la visualizzazione della territorialità all'interno della quale operano e si muovono i personaggi e le loro storie. Anche se spesso ambientati in contesti urbani e metropolitani all'apparenza facilmente riconoscibili ed identificabili dal lettore, manca infatti in questi romanzi un riferimento direi quasi rassicurante a quei landmarks architettonici e urbanistici che dovrebbero invece contraddistinguere e avvalorare emblematicamente i paesaggi e la mappatura della tradizione storico-culturale dell'identità nazionale italiana. Mi chiedo allora che tipo di implicazioni politico-letterarie possano scaturire da questa deliberata invisibilità dei landscapes ufficiali e da una esclusione dell'altro dai paesaggi urbani all'interno dei quali si muovono incerti ed esitanti questi cittadini stranieri protagonisti della scrittura migrante. Se, infatti, "la definizione dei codici di inclusione all'interno dello spazio della cittadinanza, e contestualmente la regolazione dei meccanismi di esclusione, sono le grandi questioni che lo Stato è quotidianamente costretto a 'pensare' e a ripensare per via della presenza di 'stranieri' sul suo territorio" (Mezzadra 2000: 136), allora forse non è più possi- 
bile prescindere dall'importanza e dalle implicazioni destrutturanti che categorie come appunto quelle di invisibilità ed esclusione possono avere all'interno di un'elaborazione critico-teorica e politico-culturale del concetto stesso di cittadinanza e del suo rapporto con i movimenti migratori. Quali potenziali fattori destabilizzanti della canonicità letteraria e quali forme di resistenza politica emergono allora da questi tentativi di (ri)leggere l'uniformità impermeabile del territorio nazionale proprio spostando il punto di vista interpretativo di quegli spazi pubblici e quindi spiazzando l'orizzonte d'attesa di chi legge e che in quegli spazi vorrebbe riconoscere e validare in un qualche modo il proprio senso di appartenenza identitaria?

In questo ambito vorrei fare un breve riferimento ad un romanzo, Scontro di civiltà per un ascensore in Piazza Vittorio, vincitore del premio Flaiano e pubblicato in Italia nel 2006 dell'autore italo-algerino Amara Lakhous, proprio perché a mio avviso in questo testo, ambientato interamente a Roma, assistiamo ad una provocatoria deterritorializzazione della spazialità del paesaggio urbano, attraverso una voluta decostruzione del luogo per eccellenza simbolo imprescindibile dello stato nazione italiano, la capitale, e ancor più specificatamente piazza Vittorio, situata nel cuore del rione Esquilino, luogo fortemente connotante dell'identità romana e della sua tradizione storico-urbanistica, ma allo stesso tempo spazio multietnico e per questo frammentario ed ibrido, pluricentrico e polimorfico, contraddistinto oggi dalla presenza imprescindibile di migranti provenienti un po' da tutte le parti del mondo ${ }^{1}$.

La trama, strutturata essenzialmente secondo i dettami stilistico-narrativi di un noir, perché apparentemente funzionale all'indagine dell'omicidio di un personaggio, il Gladiatore, avvenuto appunto all'interno dell'ascensore di un condominio di piazza Vittorio, serve in realtà a dar voce ad una contrastante e spesso contraddittoria serie di letture e visualizzazioni della città stessa attraverso la coralità di diversi punti di vista offerti in ogni singolo capitolo dai personaggi che abitano nel quartiere e vorrebbero raccontare la propria verità sull'accaduto ${ }^{2}$. A questa frammentarietà di percezioni paesaggistiche e dell'evolversi della vicenda narrata, fa da cornice la voce, anzi l'ululato di Amedeo/Ahmed, quasi schizofrenicamente scisso tra il suo essere metà italiano e metà straniero, protagonista dall'identità perciò ambigua e non facilmente catalogabile, e forse implicito portavoce del messaggio autoriale, proprio perché riferimento simbolico imprescindibile del bisogno impellente di raccontarsi e di comunicare in qualche modo il proprio disperato desiderio di verità: "Cos’è il silenzio? È utile parlare? Ci sono altri modi per dire la verità senza muovere le labbra? [...] Chi possiede la verità? Anzi, cos’è la verità? La verità si dice con le parole? [...] Oggi il mio odio per la verità è aumentato e la mia passione per l'ululato è cresciuta. Ululerò il resto della notte [...] e so che il mio ululato

1 E Lakhous ritorna su questa frammentarietà multietnica che in qualche modo deterritorializza inevitabilmente la spazialità urbana, in un altro romanzo ambientato anch'esso a Roma dove appunto il protagonista, proprio attraverso il suo vagabondare per i quartieri della capitale, esprime il bisogno di familiarizzare con quelle comunità ibride e straniere che contraddistinguono l'Italia del futuro: "Vado a zonzo per un'oretta e mezza come un vagabondo senza meta. Mi faccio piazza della Radio e ponte Marconi avanti e dietro. Voglio familiarizzare subito con il quartiere. Osservo attentamente le facciate dei palazzi, la varietà è impressionante, come le facce delle persone che mi passano davanti. Ci sono fisionomie di tutti i tipi: giovani neri e asiatici che vendono merce contraffatta sui marciapiedi, bambini arabi che passeggiano con il papà e la mamma col velo, fimmini rom con gonne lunghe che chiedono l'elemosina. Insomma, sono nell'Italia del futuro, come dicono i sociologi!" (Lakhous 2010: 12).

2 Non a caso il romanzo si apre con una citazione di Sciascia dal Giorno della civetta che fa esplicito riferimento proprio alla questione della verità: "La verità è nel fondo di un pozzo: lei guarda in un pozzo e vede il sole o la luna; ma se si butta giù non c’è più né sole né luna, c’è la verità” (Lakhous 2006). 
non lo ascolterà nessuno. Affiderò a questo piccolo registratore il mio incessante ululato, poi mi consolerò ascoltandolo" (Lakhous 2006: 28-9). E di questa ambiguità identitaria di fondo che caratterizza provocatoriamente il protagonista, costantemente in bilico tra il suo sentirsi per certi versi italiano ed il suo imprescindibile bisogno di affermare allo stesso tempo la propria diversità ed il proprio essere dignitosamente altro, sono consapevoli un po' tutti i personaggi stranieri del romanzo ${ }^{3}$ perché anch'essi irrimediabilmente e problematicamente consci della difficoltà culturali ed esistenziali, ma anche e soprattutto giuridiche e politicosociali, di una definizione, non semplicistica e totalizzante, ma necessariamente complessa e mutevole, del concetto stesso di cittadinanza:

È inutile insistere su questa domanda: Amedeo è italiano? Qualsiasi risposta non risolverà il problema. Ma poi chi è italiano? Chi è nato in Italia, ha passaporto italiano, carta d'identità, conosce bene la lingua, porta un nome italiano e risiede in Italia? Come vedete la questione è molto complessa. Non dico che Amedeo è un enigma. Piuttosto è come una poesia di Omar Khayyam, ti ci vuole una vita per comprenderne il significato, e solo allora il cuore si aprirà al mondo e le lacrime ti riscalderanno le guance fredde. (Lakhous 2006: 14-5)

Ecco allora perché l'ascensore diventa, già nel titolo stesso del romanzo, metafora antropologica imprescindibile di uno scontro di civiltà e quindi sinonimo di una profonda crisi identitaria ed ontologica, ma anche di uno spiazzamento visivo della centralità storico-paesaggistica di piazza Vittorio. Il microcosmo claustrofobico e soffocante dell'ascensore assurge così, proprio per il suo essere spazio limitato e all'apparenza insignificante ma in realtà luogo di incontro/ scontro tra gli abitanti del condominio obbligati a condividerlo e perciò luogo di confronto problematico tra le culture di cui essi stessi sono portavoce, a "metafora dell'Italia contemporanea e delle difficoltà sulla strada della convivenza” (Negro - Grazia 2014: 2).

Al valore ufficiale e al macroscopico peso storico e semantico del nome piazza Vittorio ${ }^{4}$ spazio monumentale umbertino e piemontese realizzato nel 1870 per celebrare l'avvenuta proclamazione di Roma capitale del nuovo stato nazione italiano - si sostituisce così, come focus narrativo, l'apparentemente insignificante microscopico spazio ascensoriale dove hanno luogo e da cui prendono spunto i dialoghi e le disquisizioni politico-esistenziali fra i vari personaggi del romanzo, proprio perché, come sottolinea giustamente Graziella Parati: "for many of the novel's characters the elevator represents the door to the city [...] it embodies the common

3 La questione problematica e conflittuale del nome come marchio imprescindibile di una diversità identitaria di fondo che contraddistingue irreparabilmente, a prescindere da problemi giuridici di cittadinanza, l'essere immigrato e straniero in Italia, ritorna provocatoriamente anche in Divorzio allislamica: "[...] per ogni immigrato la questione del nome è fondamentale. La prima domanda che ti fanno sempre è: come ti chiami? Se hai un nome straniero si crea immediatamente una barriera, una frontiera insuperabile fra il "noi" e il "voi". Il nome ti fa sentire subito se sei dentro o fuori, se appartieni al "noi" o al "voi" [...]. Non conta se sei nato in Italia, hai la cittadinanza italiana, parli perfettamente l'italiano eccetera eccetera [...] Diciamo che il nome è il primo marchio della nostra diversità” (Lakhous 2010: 23).

4 In effetti, sono proprio le percezioni massmediatiche ed ufficiali di questa piazza ad essere costantemente messe in discussione da quasi tutti i personaggi stranieri del romanzo. Così reagisce, ad esempio, Iqbal all'accusa rivolta contro Amedeo di essere l'autore dell'omicidio del Gladiatore: "Il signor Amedeo è un ricercato? Non posso credere a questa accusa [...] Io non mi fido dei giornalisti della tv, perché cercano sempre lo scandalo e ingigantiscono tutti i problemi. Quando sento quello che si dice di brutto su piazza Vittorio mi viene un dubbio: mi chiedo se davvero stiano parlando dello stesso posto dove vivo da dieci anni oppure del Bronx che vediamo nei film polizieschi” (Lakhous 2006: 47). 
rethoric used in political discourses on migration [...] allowing and disallawing movement and access" (2010: 436). Ecco, ad esempio, in quali termini, Parviz, il personaggio iraniano, si relaziona all'utilizzo personale dell'ascensore trovando in esso un'occasione per meditare sulla sua condizione di esiliato:

La vecchia Benedetta è la portiera del palazzo dove vive Amedeo a piazza Vittorio. Questa maledetta ha il vizio di nascondersi dietro l'ascensore, pronta a litigare con qualsiasi persona voglia usarlo. Io adoro l'ascensore, lo uso non per pigrizia ma per meditare. Premi il pulsante senza nessuno sforzo, vai su o scendi giù, potrebbe guastarsi mentre sei dentro. È esattamente come la vita, piena di guasti. Ora sei su, ora sei giù. Ero su [...] in paradiso [...] a Shiraz, felice con mia moglie e i miei figli, mentre adesso sono giù [...] nell'inferno, soffro di nostalgia. L'ascensore è uno strumento di meditazione. (Lakhous 2006: 16)

E se nello spazio angusto dell'ascensore Parviz riesce in qualche modo a ritrovare una momentanea ed autonoma zona di sottrazione dalle altrimenti alienanti logiche e dinamiche del suo essere straniero e migrante all'interno di una territorialità nazionale ignara dei suoi bisogni e della sua presenza, diverso è invece il suo rapportarsi ai luoghi pubblici detentori di un paesaggio urbano simbolo indiscutibile appunto di quella stessa identità territoriale. Ecco allora che il sia pur sfuggente e marginale riferimento a piazza Santa Maria Maggiore subisce, proprio a causa della sua scomoda ed inquietante presenza, una provocatoria deterritorializzazione perché filtrato non solo tramite la visione riconciliante di un'italianità condivisa o la celebrazione turistica e spettacolare del suo carattere monumentale, ma attraverso la sua sofferta e drammatica condizione di rifugiato a cui, tra l'altro, la polizia si ostina a non voler concedere il permesso di soggiorno: ${ }^{5}$

Piango molto e bevo ancora di più per dimenticare le disgrazie che mi sono capitate. Ho preso l'abitudine di sedermi tutti i giorni vicino alla fontana di fronte all'ingresso della chiesa di Santa Maria Maggiore per dare il mangime ai piccioni o per piangere [...] da oggi in poi nessuno si accorgerà di me quando piangerò e berrò a piazza Santa Maria Maggiore. Chi mi toglierà dalle mani la bottiglia di Chianti? Penso seriamente di andarmene [...] Roma, senza Amedeo, non vale nulla. È come un piatto persiano senza le spezie! (Lakhous 2006: 17-25)

5 È importante sottolineare, tra l'altro, come il riferimento al permesso di soggiorno costituisca quasi un leitmotiv nellevolversi della vicenda narrata proprio perché elemento imprescindibile del vissuto dei personaggi migranti e delle loro storie quotidiane di sopravvivenza. Così si esprime, ad esempio, Maria Cristina Gonzales, la badante peruviana: "Vorrei essere tranquilla ma non ho nemmeno i documenti. Sono come una barca con le vele distrutte, sottomessa alla volontà delle rocce e delle onde. Se avessi il permesso di soggiorno non permetterei alla portiera napoletana di prendermi in giro e di offendermi [...] Ho paura della portiera perché potrebbe denunciarmi alla polizia. Non ho il permesso di soggiorno, e se cadessi nelle loro mani non sarebbero indulgenti con me e in un batter d'occhio mi ritroverei all'aeroporto di Lima, tornerei nell'inferno della povertà. Non voglio tornare in Perù prima di aver realizzato il mio sogno di una casa, di un marito e di bambini" (Lakhous 2006: 67). A questo proposito, vedi anche Lakhous (2010: 11 e 73-4). 
La rinomata piazza romana acquista perciò una valenza semantica altra proprio perché vissuta e decostruita attraverso l'esperienza personale di Parviz 6 , che, con i suoi problemi e suoi bisogni, il suo pianto e la sua "adorazione per i piccioni" e il "grande piacere" che prova a "dar loro da mangiare" (Lakhous 2006: 24), obbliga in un certo senso chi legge a ripensare luoghi a lui/lei familiari e a (ri)stabilire un rapporto di proximity con l'ospite, lo straniero: "il divenire fuori luogo delle scritture migranti produce [cosi] una contestazione dei confini [...] una potente narrazione che trasforma gli spazi urbani [...] mettendo in questione il concetto stesso di cittadinanza, mostrando la sua evanescenza" (De Robertis 2008: 218). L'invisibilità del personaggio straniero, il suo essere figura spettrale scomoda e per questo necessariamente relegabile ai margini o addirittura utopisticamente al di fuori di una comunità istituzionalizzata e perciò garante imprescindibile dei propri diritti di cittadinanza, diventa necessariamente elemento destrutturante e potenzialmente nocivo all'interno della rappresentazione spettacolare e autogratificante dell'identità nazionale: "tanto più in un'epoca in cui ogni configurazione 'identitaria' [...] appare investita da molteplici tensioni, circostanza che asseconda la diffusione di tendenze alla chiusura difensiva e reattiva all'interno di 'piccole patrie' più o meno apertamente razziste" (Mezzadra 2000: 137).

Lo sconfinamento rizomatico a cui viene costantemente sottoposta la mappatura della città tramite la deterritorializzazione multiculturale a cui la espongono i personaggi migranti della comunità di piazza Vittorio, permette quindi una messa in discussione non solo della rigidità inattaccabile e statuaria dei paesaggi urbani e delle sue geografie, ma anche e soprattutto una sovversiva e politicamente destabilizzante rilettura del concetto stesso di identità nazionale, proprio perché questo insieme di storie spezzate delle quali si compone il romanzo, "disseminate per via dei movimenti migratori all'interno delle singole storie nazionali, ne disturbano la narrazione lineare e scardinano la temporalità omogenea dell'ipotetica comunità nazionale" (Mezzadra 2000: 142). Non è un caso che tutte le temporanee dislocazioni spaziali che caratterizzano i movimenti e i vagabondaggi ${ }^{7}$ dei personaggi, dentro e fuori del microcosmo condominiale di piazza Vittorio, avvengano quasi sempre in luoghi pubblici all'apparenza facilmente catalogabili e perciò immediatamente riconoscibili, ma che determinano allo stesso tempo inequivocabilmente una sorta di "scomposizione prismatica" (Mezzadra 2000: 139) di quella stessa mappatura urbana di cui sono appunto simbolo imprescindibile.

\footnotetext{
6 Lakhous ritorna significativamente su questa contaminazione necessaria e destabilizzante di una rappresentazione pubblica e monumentale dei luoghi emblematici dell'identità nazionale in Divorzio all'islamica, dove appunto Sofia, personaggio anch'esso in bilico rispetto alla sua appartenenza identitaria, trasforma la spettacolarità oggettiva e condivisa della fontana di Trevi in una visione onirica e perciò fortemente soggettiva, perturbante e evanescente della stessa spazialità: "Sono seduta di fronte alla fontana di Trevi [...] Vedo la biondona (quella della Dolce vita) dentro la fontana, sotto la cascata d'acqua. Di colpo inizia a gridare: "Marcello, come here!". Io rimango a osservare, ma sono accecata dalla gelosia. Marcello Mastroianni è seduto a prendersi un caffè e non si muove [...] Dopo un po' si avvicina a me. E quando i nostri sguardi si incrociano mi accorgo che Marcello ha il volto del ragazzo del fazzoletto (l'arabo senza nome) che ho incontrato prima al Little Cairo [...] mi abbraccia con estrema dolcezza. Sono così felice. Ma il sogno finisce e io mi risveglio bruscamente" (2010: 100).
}

7 Il vagare nomadico e nostalgico attraverso territorialità reali ed immaginarie, italiane o straniere, contraddistingue infatti i percorsi e le vicende esistenziali dei personaggi migranti; ecco, ad esempio, in quali termini Parviz riflette amaramente sulla sua condizione di esiliato costretto a vivere lontano dalla propria famiglia: "Quando ricordo i miei bambini: Shadi, Said, Surab, Omar e mia moglie Zeinab mi rattristo molto. Dove saranno adesso? Staranno vagando chissà dove. Vorrei baciarli e abbracciarli tutti. Solo le lacrime [...] spengono il fuoco della nostalgia" (Lakhous 2006: 17). 
In un altro momento fortemente emblematico di questo processo di ibridazione della territorialità nazionale è infatti proprio la badante peruviana, Maria Cristina Gonzales, a spingersi fuori del condominio dove accudisce Rosa, un'anziana signora paralitica, e ad accompagnare il lettore in un percorso solitario di (ri)scoperta della città che si conclude proprio davanti alla stazione Termini, luogo comunemente ed emblematicamente vissuto come momento di passaggio e transitorietà, ma in realtà per lei momento emozionale indispensabile di aggregazione comunitaria amorosa ${ }^{8}$ tra i suoi connazionali:

Faccio di tutto per non perdere minuti preziosi, metto a punto un programma ricco di impegni, ma ogni volta faccio la stessa cosa: vado alla stazione Termini dove si incontrano gli immigrati peruviani. I loro volti soddisfano la sete dei miei occhi e le loro parole riscaldano le mie orecchie fredde. Mi sembra di tornare a casa, a Lima. Saluto e bacio tutti anche se alcuni non li ho mai visti prima, poi mi siedo sul marciapiede e divoro i cibi peruviani, il riso con pollo e il lomo saltado e il sibice. Parlo per ore, parlo più di quanto ascolto, per questo mi chiamano Maria Cristina la chiacchierona. (Lakhous 2006: 66)

Ancora una volta quindi il paesaggio si trasforma davanti ai nostri occhi, perde la sua prevedibile e uniforme spettacolarità ed acquista proprio a causa di questo flusso - "the modern world is in a state of flux and turbulence", sostiene appunto Nikos Papastergiadis, secondo il quale, in effetti, "migration, in its endless motion, surrounds and pervades almost all aspects of contemporary society" (Papastergiadis - Nikos 2000: 1) - di soggetti sociali ibridi in continuo movimento e divenire identitario, una sua destabilizzante liquidità spazio-temporale in grado di sovvertire potenzialmente predefinite costruzioni politico-sociali e culturali di quello stesso luogo definite dal progetto uniformante ed egemonico dello stato nazione italiano. Si tratta, in altre parole, della possibilità di rendere frammentario e ambivalente precisamente quello stesso territorio, i suoi confini e le sue regolamentazioni, di (ri)disegnare "una nuova cartografia" della cittadinanza globale attraverso forme autonome di aggregazioni costituenti del comune e quindi di far sì che "lo sguardo eccentrico del migrante rivolto agli spazi urbani di arrivo [funzioni] come meccanismo di straniamento non solo nella rappresentazione dei nostri spazi di vita ma anche all'interno dei discorsi che la modernità aveva codificato intorno ai propri spazi metropolitani” (Mengozzi 2008: 3).

Se dunque "space is a dynamic field in which identities are in a constant state of interaction" (Papastergiadis - Nikos 2000: 4), allora forse si potrebbe pensare alla scrittura migrante come ad un luogo di sperimentazione innovativa all'interno del quale le spazialità etniche e multiculturali possano trovare una loro necessaria ed imprescindibile visibilità, dove realtà politico-culturali ed identitarie certamente marginali e per questo costantemente in crisi, riescono

8 Ed è, infatti, la stessa Maria Cristina a sottolineare drammaticamente la propria voglia ed il bisogno disperato di sentirsi viva: "mi allontano in silenzio dagli sguardi e sotto l'ala della notte me ne vado con un giovane che mi assomiglia in tutto. Ognuno di noi svuota nel corpo dell'altro la propria voglia, speranza, angoscia, paura, tristezza, rabbia, odio e delusione, e questo lo facciamo in fretta come gli animali che hanno paura di perdere la stagione della fertilità. Ci stendiamo su una panchina isolata o su pagine di giornali sparsi per terra" (Lakhous 2006: 66).

9 Scrivono Hardt e Negri: "Il diritto universale di controllare i propri movimenti è l'istanza radicale della moltitudine per una cittadinanza globale [...] La cittadinanza globale è il potere con cui la moltitudine si riappropria del controllo sullo spazio e con cui disegna una nuova cartografia" (2000: 370). 
tuttavia a materializzarsi attraverso "forme residuali [...] spazi deboli [...], apparizioni rigorosamente temporanee, addirittura volatili, evanescenti, effimere" (Papotti 2007: 392). (Ri)pensare insomma a nuove modalità e pratiche politiche attraverso le quali "those who have no part" (Rancière 1999: 77), direbbe Rancière, possano "interrompere la temporalità del consenso" (Rancière 1999: 7-9 in Chignola e Mezzadra 2012: 4) e partecipare ad un processo necessario di delegittimazione di specifiche strategie di controllo portate avanti dalla sovranità nazionale e di trasformazione radicale del suo tessuto identitario geopolitico, socio-culturale ed ideologico. In questa prospettiva si potrebbe quindi arrivare ad un ripensamento necessario del concetto stesso di cittadinanza e del valore politico e giuridico che ad esso viene attribuito soprattutto in rapporto alle pratiche di resistenza e di insubordinazione sostenute da stranieri, clandestini e migranti all'interno della territorialità nazionale. Ed è appunto in questo senso che è anche possibile ripensare al significato stesso di cittadinanza non come ad un qualcosa di rigido e predefinito, ma invece, come ad una institution in flux (Mezzadra 2010), una insurgent e transnational citizenship (Balibar 2006); intraprendere, in altre parole, un percorso politico-culturale che riguardi la rivisitazione del rapporto tra diritti di cittadinanza e movimenti migratori e quindi la messa in discussione radicale di paradigmi concettuali quali integrazione, esclusione e appartenenza sviscerandone proprio la loro intrinseca componente ambigua e contraddittoria alla luce soprattutto di un inarrestabile ed ineluttabile confondersi e sfumarsi di una separazione netta tra confini, frontiere e territorialità nazionali. Ed è proprio in questo senso che diventa allora possibile pensare ad una sorta di autonomy of migration, un approccio critico-teorico e politico che riconosca cioè il dissidio, la fuga e l'auto-esclusione come segnali imprescindibili di insubordinazione e di rifiuto da parte del migrante che non vuole sentirsi necessariamente né dentro né fuori le mappature geopolitiche e le codificazioni territoriali dettate dalle logiche sovrane e nazionali e che valorizzi perciò, ci ricorda ancora Sandro Mezzadra, "the importance of practices and claims of those who are not necessarily citizens in juridical terms for the development of an understanding of the transformation of the legal framework of citizenship itself" (Mezzadra 2010: 1). La condizione di invisibilità e di non-appartenenza che caratterizza spesso questa moltitudine di soggetti stranieri costantemente dislocati e delegittimizzati dei loro diritti di cittadini senza cittadinanza e della loro dignità di esseri umani rappresenta quindi, a mio avviso, un elemento peculiare potenzialmente sovversivo e politicamente necessario proprio della loro stessa condizione di migranti. È infatti in virtù del loro essere in continuo movimento che questi soggetti sociali possono sottrarsi temporaneamente al controllo ed alle regolamentazioni della sovranità nazionale e divenire quindi impercettibili - becoming imperceptible, come direbbero Deleuze e Guattari; anche se questo vagabondare nomadico, non bisogna mai dimenticarlo, si esplica spesso attraverso l'inevitabile bagaglio di sofferenza e dolore che la loro drammatica condizione esistenziale e sociale comporta, e dal quale in ogni caso non è possibile prescindere per escluderne qualsiasi banale e pericolosa idealizzazione romantica. Si tratta, in altre parole, di istituire, secondo l'intuizione di Papadopulous e Tsianos, dei mobile commons e cioè entità autonome di sopravvivenza che possano operare eludendo momentaneamente le strategie di controllo e di regolamentazione poliziesca portate avanti dagli stati-nazione e diventare così "migrants' resource and path for surviving the pressures of sovereignty and capitalist exploitation [...] The mobile commons is the ontology of transmigration" (Papadopoulus - Vassilis 2013: 192). 
Ecco allora perché nel romanzo diventano improponibili e pericolose, perché xenofobiche e deliranti, le prese di posizioni di chi come, Antonio Marini, il professore di origini milanesi obbligato anch'egli, suo malgrado, a condividere questo caotico spazio condominiale, vorrebbe appunto sintetizzare in poche, schematiche parole, semplificandone e appiattendone così qualsiasi diversità e complessità di fondo, la sua inattaccabile visione della "vera" Roma e più in generale dell'intera realtà urbana del sud d'Italia:

Roma! La città eterna! La bella Roma! Roma amor! No, mi dispiace, io non guardo Roma con gli occhi del turista che viene per una settimana o due, fa un giro a piazza Navona, a piazza di Spagna, a Fontana di Trevi, scatta qualche foto ricordo, mangia la pizza e gli spaghetti e poi ritorna nel suo paese. Io non vivo nel paradiso dei turisti, ma nell'inferno del caos! Per me non c’è differenza tra Roma e le città del sud, Napoli, Palermo, Bari e Siracusa. Roma è una città del sud e non ha niente a che fare con città come Milano, Torino e Firenze. La gente di Roma è pigra, questa è l'evidente verità. (Lakhous 2006: 74)

Ed è appunto in opposizione politica a queste percezioni sicuramente inaccettabili e fuorvianti della territorialità nazionale, metafora emblematica e significativa dell'uso di un linguaggio egemonico funzionale alla celebrazione di un'appartenenza identitaria rigida e categorica e perciò impermeabile ed incontaminata dalla presenza dell'Altro, che Lakhous offre al suo lettore, attraverso una scrittura caratterizzata invece da un linguaggio "minore" e marginale ma certamente capace di offrire, proprio per il suo essere invece fortemente contaminato dalla polifonia di voci di queste figure migranti, una visione più porosa della spazialità urbana e quindi, in qualche modo, di immaginare nuove comunità transnazionali e nuove abitabilità territoriali in divenire.

Si tratta, in altre parole, di un tentativo potenzialmente destrutturante di rielaborazione radicale della nostra stessa percezione dello spazio e di un bisogno impellente di (ri)pensare così, proprio nell'implicita tensione che caratterizza oggi l'imprescindibile conflittualità tra locale $\mathrm{e}$ globale, nazionale e transnazionale, material e virtual landscape, linguaggi altri che possano rappresentare e descrivere appunto le trasformazioni culturali in atto all'interno di questo nuovo e per certi versi ancora in fieri paesaggio geopolitico: una sorta di ethnoscape, per usare la definizione di Arjun Appadurai, e cioè di un "landscape of persons who constitute the shifting world in which we live: tourists, immigrants, refugees, exiles, guest workers, and other moving groups and individuals constitute an essential feature of the world and appear to affect the politics of (and between) nations to a hitherto unprecedented degree" (Appadurai 1996: 33).

Forse quindi sono proprio questi ethnoscapes che Amara Lakhous ci invita a esplorare e indagare attraverso la sua scrittura: luoghi all'apparenza marginali, irriconoscibili e conflittuali ${ }^{10}$,

10 E lo stesso Lakhous sembra essere certamente consapevole nella sua scrittura di questa contraddittorietà e conflittualità di fondo che contraddistingue i luoghi marginali di aggregazione delle comunità di migranti in terra straniera. Vedi, ad esempio, come l'autore rappresenta le dinamiche socio-culturali ed emotive che, in Divorzio all'islamica, caratterizzano la quotidianità di un call center nel centro di Roma: "Il Little Cairo è affollato, molti clienti non si limitano come me a telefonare, ma si fermano a guardare Madame al-Jazeera. Il televisore serve ad attirare i clienti e a farli sentire a casa. Purtroppo sono in tanti a cascarci. Non so come fanno a stare ore e ore a seguire le notizie di attentati, bombe, kamikaze, guerre, morte. È un bombardamento mediatico quotidiano. Un autentico doping della mente e della memoria. Poveri immigrati, ogni giorno assorbono una quantità impressionante di negatività, rischiando di diventare dei malati, dei drogati” (2010: 145). 
quasi evanescenti ed invisibili, ma con i quali è necessario stabilire comunque un rapporto di familiarizzazione e di integrazione reciproca per cercare di comprenderne le sostanziali differenze ed ibridazioni di fondo e quindi la loro inarrestabile e necessaria metamorfosi identitaria: "Even if migration starts sometimes as a form of dislocation (forced by poverty, patriarchal exploitation, war, famine), its target is not relocation but the active transformation of social space [...] Nomadic motion is not about movement but about the appropriation and remaking of space" (Papadopoulos - Tsianos 2007: 224) ${ }^{11}$.

Sebbene, infatti, il romanzo si concluda con le parole rassicuranti del Commissario Bettarini - incaricato di indagare appunto sull'omicidio del Gladiatore e per il quale la soluzione del giallo è anche il risultato del suo conoscere "abbastanza bene questa zona. Ho passato molti anni al commissariato dell'Esquilino - continua infatti in tono rassicurante - e ho avuto la possibilità di avvicinarmi ai problemi dei residenti di piazza Vittorio" (Lakhous 2006: 123), il lettore è forse allo stesso tempo consapevole che alla fine della vicenda narrata non è importante in fondo risolvere il caso e scoprire, secondo i dettami del giallo tradizionale, il colpevole, ma piuttosto acquisire consapevolezza, grazie alla molteplicità fluida e frammentaria delle percezioni paesaggistiche e della presunta non-italianità linguistica ed autoriale attraverso cui viene rappresentata e (ri)scritta Roma con i suoi paesaggi urbani "minori", di un possibile ripensamento radicale, nell'era della globalizzazione e della logica neoliberista del capitalismo transnazionale, dell'ufficiosità mistificatoria e totalizzante dell'identità territoriale dello stato nazione italiano e quindi di una contaminazione e problematizzazione necessaria della nozione stessa di cittadinanza.

\section{Riferimenti bibliografici}

Appadurai, A. (1996). Modernity at Large: Cultural Dimensions of Globalization. Minneapolis: University of Minnesota Press.

Balibar, É. (2006). Strangers as Enemies: Further Reflections on the Aporias of Transnational Citizenship. Globalization Working Papers, 4, 3-20.

Chignola, S., \& Mezzadra, S. (2012). Fuori dalla pura politica. Laboratori globali della soggettività [online], 1-13. In: http://www.uninomade.org/fuori-dalla-pura-politica/\#_ftn16.

De Robertis, R. (2008). Storie fuori luogo. Migrazioni, traduzioni e riscritture in Scontro di civiltà per un ascensore a piazza Vittorio. Studi d'italianistica nell'Africa australe, 21 (1-2), 215-41.

Hardt, M., \& Negri, A. (2000). Impero. Il nuovo ordine della globalizzazione. Milano: Rizzoli. Lakhous, A. (2010). Divorzio allislamica a viale Marconi. Roma: Edizioni e/o. . (2006). Scontro di civiltà per un ascensore a piazza Vittorio. Roma: Edizioni e/o.

Mengozzi, C. (2008). Città e modernità: nuovi scenari urbani nell'immaginario della "letteratura italiana della migrazione". In: C. Gurreri, A. M. Jacopino, \& A. Quondam (Eds.), Moderno e modernità: la letteratura italiana [online]. Roma: La Sapienza Editore. In: http://www.italianisti.it/FileServices/Mengozzi\%20Chiara.pdf.

11 Scrivono, ancora a questo proposito, Papadopulous e Tsianos: "Migration makes material and psychosocial spaces porous, a Benjaminian porosity, where public and private intermingle, deviance and norm are renegotiated, zones of exploitation and justice are rearranged, formal and informal situations are reassembled" (226). 
Mezzadra, S. (2010). The Gaze of Autonomy. Capitalism, Migration and Social Struggles. UniNomade [online], September 19, 1-17. In: http://uninomade.org/the-gaze-of-autonomy-capitalism-migration-andsocial-struggles/print.

. (2000). Cittadini della frontiera e confini della cittadinanza. Per una lettura politica delle migrazioni contemporanee. aut-aut, 298, 133-53.

Negro, G. (2014). L’upupa o L’Algeria perduta: i nuclei tematici, il processo di riscrittura e la ricezione nel mondo arabo di Amara Lakhous, Kúmá/critica [online], 1-13. In: http://www.disp.let.uniroma1.it/ kuma/critica/kuma12upupa.html.

Papadopoulus, D., \& Tsianos, V. (2007). The Autonomy of Migration: The Animals of Undocumented Mobility. In: A. Hickey-Mood, \& P. Malins (Eds.), Deleuzian Encounters. Studies in Contemporary Social Issues [online] (pp. 223-35). Basingstoke: Palgrave-Macmillan. In: http://www2.leicester.ac.uk/departments/management/research/units/cppe/archiveactivities/texts/2007-Migration-DeleuzianEncountersPapadopoulos-Tsianos.PDF/view.

. (2013). After Citizenship: Autonomy of Migration, Organizational Ontology and Mobile Commons. Citizenship Studies, 17 (2), 178-96.

Papastergiadis, N. (2000). The Turbulence of Migration. Globalization, Deterritorialization and Hybridity. Cambridge: Polity Press.

Papotti, D. (2007). Fra marchi etnici e mimetismo identitario. Riflessioni sulle dinamiche di evoluzione dei paesaggi etnici in Italia a partire dal Piemonte orientale. In: P. Nodari, \& G. Rotondi, (Eds.), Verso uno spazio multiculturale? Riflessioni geografiche sullesperienza migratoria in Italia (pp. 389-401). Bologna: Pàtron Editore.

Parati, G. (2010). Where Do Migrants Live? Amara Lakhous's Scontro di civiltà per un ascensore a Piazza Vittorio. Annali d'Italianistica, 28, 431-45.

Rancière, J. (1999). Dis-agreement. Politics and Philosophy. Minneapolis: University of Minnesota Press. . (2009). Moments politiques. Interventions 1977-2009. Paris: La Fabrique. 
